\title{
First Draft Genome Assemblies of Pleochaeta shiraiana and Phyllactinia moricola, Two Tree-Parasitic Powdery Mildew Fungi with Hemiendophytic Mycelia
}

\author{
Stefan Kusch, ${ }^{1, \dagger}$ Niloofar Vaghefi, ${ }^{2}$ Susumu Takamatsu, ${ }^{3}$ Shu-Yan Liu, ${ }^{4}$ Márk Z. Németh, ${ }^{5}$ \\ Diána Seress, ${ }^{5}$ Lamprinos Frantzeskakis, ${ }^{6}$ Pin-En Chiu, ${ }^{7}$ Ralph Panstruga, ${ }^{1}$ and Levente Kiss ${ }^{2,5}$ \\ ${ }^{1}$ Unit of Plant Molecular Cell Biology, Institute for Biology I, RWTH Aachen University, Aachen, \\ Germany \\ ${ }^{2}$ Centre for Crop Health, University of Southern Queensland, Toowoomba, Australia \\ ${ }^{3}$ Laboratory of Plant Pathology, Faculty of Bioresources, Mie University, Tsu, Japan \\ ${ }^{4}$ College of Plant Protection, Jilin Agricultural University, Changchun, China \\ ${ }^{5}$ Plant Protection Institute, Centre for Agricultural Research, Eötvös Loránd Research Network (ELKH), \\ Budapest, Hungary \\ ${ }^{6}$ Bayer Crop Science, 700 W Chesterfield Pkwy W, Chesterfield, MO 63017, U.S.A. \\ ${ }^{7}$ Department of Chemistry, University of Michigan, Ann Arbor, MI, U.S.A.
}

\begin{abstract}
Powdery mildew fungi (Erysiphaceae) are widespread obligate biotrophic plant pathogens. Thus, applying genetic and omics approaches to study these fungi remains a major challenge, particularly for species with hemiendophytic mycelium. These belong to a distinct phylogenetic lineage within the family Erysiphaceae. To date, only a single draft genome assembly is available for this clade, obtained for Leveillula taurica. Here, we generated the first draft genome assemblies of Pleochaeta shiraiana and Phyllactinia moricola, two treeparasitic powdery mildew species with hemiendophytic mycelium, representing two genera that have not yet been investigated with genomics tools. The Pleochaeta shiraiana assembly was $96,769,103 \mathrm{bp}$ in length and consisted of 14,447 scaffolds, and the Phyllactinia moricola assembly was $180,382,532$ bp in length on 45,569 scaffolds. Together with the draft genome of $L$. taurica, these resources will be pivotal for understanding the molecular basis of the lifestyle of these fungi, which is unique within the family Erysiphaceae.
\end{abstract}

\section{Genome Announcement}

More than 10,000 dicot and monocot plant species, including important crops, are commonly infected by powdery mildew fungi (Erysiphaceae, Helotiales, Ascomycota) on a global scale (Braun and Cook 2012; Glawe 2008; Kiss et al. 2020). In total, there are approximately 900 species of powdery mildew fungi in 19 recognized genera (Braun and Cook 2012; Kiss et al. 2020). All of these ubiquitous fungi are obligate biotrophic plant pathogens, which means that nutrient uptake can happen only from their living host plant tissues and, thus, they cannot

\footnotetext{
${ }^{\dagger}$ Corresponding author: S. Kusch; skusch@bio1.rwth-aachen.de
}

Author contributions: Epiphytic fungal materials were collected by S.L. (Phyllactinia moricola), S.T., M.Z.N., and D.S. (Pleochaeta shiraiana). High molecular weight DNA was isolated and DNA quality control performed by L.F., S.K., and S.L. S.K. did genome assemblies, assembly quality control, and filtering. N.V. conducted gene prediction and phylogenetic analysis of nrDNA sequences. P.C. and N.V. performed the transposable element analysis. R.P. and L.K. provided reagents, materials, and resources. S.K. and L.K. drafted the manuscript, and all authors edited and proofread the manuscript.

*The $e$-Xtra logo stands for "electronic extra" and indicates that a supplementary table is published online.

The author(s) declare no conflict of interest.

Accepted for publication 12 September 2021.

$e-$ tra $^{*}$

\section{Funding}

This work was supported, in part, by a grant of the Australia-Germany Joint Research Co-Operation Scheme, and the program PPP Australia 2019 funded by the German Academic Exchange Service (Deutscher Akademischer Austauschdienst), awarded to L. Kiss and R. Panstruga. It was further supported by the German Research Foundation (Deutsche Forschungsgemeinschaft [DFG]) project number 274444799 [grant 861/14-1 awarded to R. Panstruga] in the context of the DFG-funded priority program SPP1819 "Rapid evolutionary adaptation-potential and constraints", and also by the University of Southern Queensland.

\section{Keywords}

evolution, fungal pathogens, genomic resources, genomics, host-parasite interactions, obligate biotrophs, plant pathogens 
grow or be cultured in the absence of their hosts (Panstruga and Schulze-Lefert 2002). The mycelia of most powdery mildew species are epiphytic, with haustoria (i.e., feeding structures) developed exclusively in the epidermal cells of their plant hosts (Kuhn et al. 2016; Micali et al. 2011, 2008). Species of the genera Leveillula, Queirozia, Pleochaeta, and Phyllactinia represent a distinct phylogenetic lineage within the Erysiphaceae family known as the endoparasitic group (Takamatsu 2013; Takamatsu et al. 2016). The endoparasitic group comprises, in total, approximately 85 species (Braun and Cook 2012). These powdery mildew fungi have partly endophytic (hemiendophytic) mycelia, with haustoria found exclusively in the mesophyll cells of their hosts (Zheng et al. 2013). Because their infection process, most notably the localization of haustoria, is markedly different from the rest of the members of Erysiphaceae, their effector repertoire and other molecular patterns associated with the hemiendophytic lifestyle are likely to be different from that of the epiphytically proliferating powdery mildew fungi.

Omics-based approaches can be powerful methods to understand molecular mechanisms underlying the powdery mildew lifestyle but are challenging due to the obligate biotrophic nature of these fungi (Bindschedler et al. 2016). This is particularly true for the species with hemiendophytic mycelium. So far, a single draft genome assembly is available for the entire group, that of Leveillula taurica collected from sweet pepper (Capsicum annuum) (Kusch et al. 2020). The genomes of powdery mildew fungi are generally large and gene poor compared with other ascomycetes (Barsoum et al. 2019; Mohanta and Bae 2015), coinciding with an abundance of repetitive elements (Frantzeskakis et al. 2018; Spanu et al. 2010; Wicker et al. 2013). Therefore, most powdery mildew genomes published thus far are highly fragmented (Barsoum et al. 2019; Bindschedler et al. 2016). Here, we present the first annotated draft genomes of Pleochaeta shiraiana infecting Chinese hackberry (Celtis sinensis) and Phyllactinia moricola infecting white mulberry (Morus alba), obtained by short-read next-generation DNA sequencing.

We collected powdery mildew-infected leaves of $C$. sinensis from the Mie University Campus (Tsu-shi, Mie Prefecture, Japan) in December 2017, and of M. alba in Yintai City (Shandong Province, China) in November 2019. We identified the powdery mildew species infecting C. sinensis as Pleochaeta shiraiana and the powdery mildew colonizing M. alba as Phyllactinia moricola based on morphology and the internal transcribed spacer (ITS) sequences of their nuclear ribosomal DNA (nrDNA) determined as described earlier (Kiss et al. 2006; Takamatsu et al. 2008). The Pleochaeta shiraiana specimen was deposited at Institutsbereich Geobotanik und Botanischer Garten, Martin-Luther-Universität Halle-Wittenberg, Germany, under accession number HAL3440 F, and its ITS sequence in GenBank (MZ661116). The Phyllactinia moricola specimen was deposited at Herbarium of Mycology of Jilin Agricultural University, China, under accession HMJAU-PM91933; its ITS sequence is available in GenBank (MZ541088). We collected the external part of the powdery mildew mycelium, including many fruiting bodies (chasmothecia) of both species, from leaves of their respective host plants with cellulose acetate peelings, as described before (Frantzeskakis et al. 2019). The peelings were collected in $2-\mathrm{ml}$ reaction tubes and ground to a fine powder in liquid nitrogen using metal balls $(3 \mathrm{~mm}$ in diameter) and a bead mill at $30 \mathrm{~m} / \mathrm{s}$ for $30 \mathrm{~s}$. We followed a modified protocol from Feehan et al. (2017) to isolate and purify high molecular weight DNA, as described previously (Frantzeskakis et al. 2019; Kusch et al. 2020). We assessed the quality of the DNA by agarose gel electrophoresis $(0.8 \%$ agarose, $60 \mathrm{~V})$ and measured the DNA amount using the Qubit dsDNA BR Assay Kit (Thermo Fisher Scientific, Waltham, MA, U.S.A.). Shortread DNA sequencing was conducted on a NovaSeq6000 machine by CeGaT (Tübingen, Germany) for Pleochaeta shiraiana and by GENEWIZ (Leipzig, Germany) for Phyllactinia moricola. The reads were quality and adapter trimmed using Trimmomatic v0.36 with the following settings: ILLUMINACLIP:TruSeq3-PE.fa:5:30:10 SLIDINGWINDOW:3:18 LEADING:6 TRAILING:6 MINLEN:90 (Bolger et al. 2014). We assessed the quality of the DNA sequencing data before and after trimming with FastQC v0.11.5 (Babraham Bioinformatics, Cambridge, U.K.). Subsequently, we generated draft genome assemblies for Pleochaeta shiraiana and Phyllactinia moricola using SOAPdenovo2 (Luo et al. 2012) and filtered the scaffolds based on BLASTN against the nucleotide database (updated February 2021), DNA sequencing remapping coverage, and GC content via BlobTools (Laetsch and Blaxter 2017), following the same procedure as described by Kusch et al. (2020). This pipeline filtered out viral, bacterial, and other nonfungal contaminating contigs.

The assembly of Pleochaeta shiraiana contained 14,447 scaffolds with a total length of $96,769,103$ bp after filtering (Table 1). We used Benchmarking Universal Single-Copy Ortholog (BUSCO) v5 with the ascomycota_odb10 (Simão et al. 2015), and identified 1,296 complete 
Table 1. Genome assembly statistics for Phyllactinia moricola and Pleochaeta shiraiana compared with the genomic features of Leveillula taurica

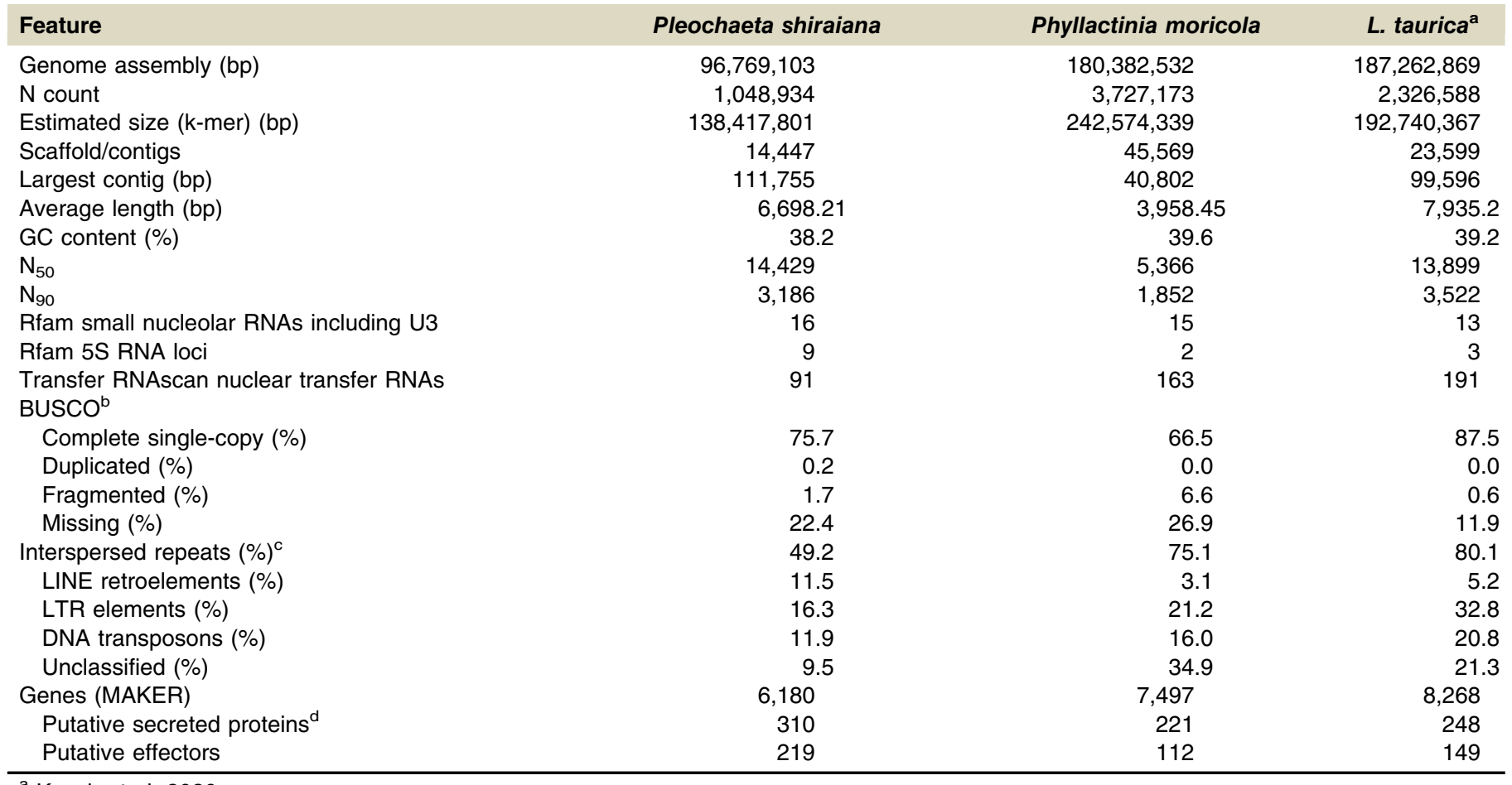

a Kusch et al 2020 .

${ }^{\mathrm{b}}$ Using the database for ascomycete core genes (ascomycota_odb10) for Benchmarking Universal Single-Copy Orthologs (BUSCOs).

${ }^{c}$ LINE = long interspersed nuclear element and LTR $=$ long terminal repeat.

d Identified using SignalP5.0; transmembrane domain proteins were filtered by TMHMM-2.0c.

and 29 fragmented of the 1,706 core ascomycete genes (77.6\%). Thus, the assembly is approximately $8 \%$ less complete than the assembly of $L$. taurica (Kusch et al. 2020), which is the only genomic resource available thus far for powdery mildew fungi with hemiendophytic mycelium. Genome size calculation via jellyfish 30-mers (Marçais and Kingsford 2011) estimated the $P$. shiraiana genome size to be approximately $138.4 \mathrm{Mbp}$. The Phyllactinia moricola assembly consisted of 45,569 scaffolds totaling 180,382,532 bp in length and had 1,135 complete and 113 fragmented $(73.1 \%)$ core ascomycete genes (Table 1$)$. The genome size was estimated to be 242.6 Mbp, representing one of the largest known powdery mildew genomes. By comparison, the two largest genome assemblies to date are from Erysiphe alphitoides at $220 \mathrm{Mbp}$ and L. taurica with $187 \mathrm{Mbp}$ (Dutech et al. 2020; Kusch et al. 2020). The new genome assemblies are publicly available at NCBI GenBank under accessions JAHYSP000000000 (Pleochaeta shiraiana) and JAHYSQ000000000 (Phyllactinia moricola). We identified 15 small nucleolar RNAs (snoRNAs) in both fungi: the fungal snoRNA U3 in Pleochaeta shiraiana but not Phyllactinia moricola and the spliceosomal RNAs U1, U2, U4, and U6 by Rfam searches (Table 1). The U5 spliceosomal RNA was not detectable in the assemblies even at an E value threshold of 0.01 . We further identified the large and small subunits of the nrDNA on contig C475265 of Pleochaeta shiraiana and on contig C733007 of Phyllactinia moricola, nine 5S ribosomal RNA loci in Pleochaeta shiraiana, and two in Phyllactinia moricola. Pleochaeta shiraiana had 91 transfer RNAs (tRNAs) and Phyllactinia moricola had 163 tRNAs decoding all 20 standard amino acids based on tRNAscan-SE (Table 1) (Chan et al. 2021). In the absence of expressed sequence tags, whole-transcriptome shotgun sequencing (RNA sequencing) data, protein evidence for Pleochaeta shiraiana and Phyllactinia moricola, ab initio genome annotations were conducted in MAKER pipeline v3.01 (Holt and Yandell 2011) using AUGUSTUS v3.3.3 (Stanke and Waack 2003) trained for L. taurica (Kusch et al. 2020), which is the closest relative of Pleochaeta shiraiana and Phyllactinia moricola with available transcriptome data. This MAKER pipeline identified 7,497 and 6,180 coding sequences in the Phyllactinia moricola and Pleochaeta shiraiana genomes, respectively. These annotations likely underestimate the number of coding genes due to lower recovery of the total genomic sequence ( 73 to $76 \%$ calculated completeness) in these assemblies. Nonetheless, these values are comparable with other powdery mildews, which carry fewer genes than other ascomycetes (Frantzeskakis et al. 2018; Jones et al. 2014; Liang et al. 
2018). Next, we employed SignalP5.0 (Almagro Armenteros et al. 2019) and filtered out transmembrane proteins via TMHMM v2.0c to predict secreted proteins in Phyllactinia moricola $(n=221)$, Pleochaeta shiraiana $(n=310)$, and L. taurica $(n=248)$ (Table 1). EffectorP3.0 (Sperschneider and Dodds 2021) predicted 112 effectors in Phyllactinia moricola, 219 in Pleochaeta shiraiana, and 149 in L. taurica, which is similar to other dicot-infecting powdery mildew species (Jones et al. 2014; Liang et al. 2018). We used RepeatModeler to discover repetitive sequences in the genomes of Phyllactinia moricola, Pleochaeta shiraiana, and L. taurica, and RepeatMasker v4.0.9 (Smit et al. 2016) to map repeats to their respective genomes. In all, 47,137,141 bp (49.2\%) in Pleochaeta shiraiana, 133,135,259 bp (75.1\%) in Phyllactinia moricola, and $148,336,729$ bp $(80.1 \%)$ in $L$. taurica were covered by repeats and transposons (Table 1$)$. The majority of these elements were long terminal repeat (LTR) retroelements and DNA transposons (almost exclusively Tc1-IS630-Pogo); however, $>15 \%$ were unclassified repeats. This architecture is similar to the genomes of different formae speciales of the cereal powdery mildew pathogen Blumeria graminis, which consist of $>70 \%$ repetitive elements (Frantzeskakis et al. 2018; Müller et al. 2019, 2021). Interestingly, DNA transposons, particularly of the type Tc1IS630-Pogo, were highly abundant in the two new draft genome assemblies and the draft genome of $L$. taurica (11.9 to $20.8 \%$ ) (Table 1 ). This is markedly different from $B$. graminis, whose genomes are dominated by long interspersed nuclear element and LTR retrotransposons (Frantzeskakis et al. 2018; Spanu et al. 2010; Wicker et al. 2013). The characteristics and the fragmentary nature of the short-read-based assemblies of the highly repetitive Pleochaeta shiraiana and Phyllactinia moricola genomes are largely consistent with the genomic features of epiphytically proliferating powdery mildew fungi (Jones et al. 2014; Liang et al. 2018; Wu et al. 2018).

Then, we retrieved nucleotide sequences of the ITS, 18S, and $28 \mathrm{~S}$ regions of the nrDNA from 18 reference powdery mildew specimens from NCBI GenBank (Supplementary Table S1) to include representative species of all of the genera with hemiendophytic mycelium, and representatives of epiphytic species, which were phylogenetically closely related to the hemiendophytic lineage in previous analyses (Bradshaw and Tobin 2020; Jin et al. 2021; Kiss et al. 2020; Marmolejo et al. 2018). The $18 \mathrm{~S}$ and $28 \mathrm{~S}$ sequences of the nrDNA region in Pleochaeta shiraiana strain HAL $3440 \mathrm{~F}$ and $18 \mathrm{~S}$ sequence of Phyllactinia moricola strain HMJAUPM91933 sequenced in this study as well as ITS, 18S, and 28 S sequences of $L$. taurica strain HNHM-MYC-006405 sequenced by Kusch et al. (2020) were extracted from their respective genome assemblies (Supplementary Table S1). We excluded the sequences of the ITS1 and ITS2 regions from the analysis because they were too variable to allow an unambiguous alignment. Multisequence alignments were constructed in MAFFT v7.450 (Katoh and Standley 2013). The concatenated alignment included a total of 1,089 nucleotide sequences (5.8S, 154 bp; 18S, $589 \mathrm{bp}$; and 28S, $587 \mathrm{bp}$ ). Maximum-likelihood analysis of the concatenated alignment was conducted using RAxML v8 (Stamatakis 2014) in Geneious Prime v2021.1.1 (https://www. geneious.com) based on the GTR substitution model applied to individual partitions and 1,000 bootstrap replicates. The tree was rooted to Parauncinula polyspora MUMH 197. Bayesian analysis of the same alignment was conducted in MrBayes v3.2.4 (Ronquist and Huelsenbeck 2003) based on SYM, K80+I, and SYM+I+G, nucleotide substitutions models selected by MrModeltest v2.3 (Nylander 2004) for 5.8S, 18S, and 28S loci, respectively. Two Markov Chain Monte Carlo chains were run and one tree was saved per 100 generations. The run was ended when the standard deviation of split frequencies reached $<0.01$. The $50 \%$ majority-rule consensus tree was generated after $25 \%$ burn-in of the saved trees. The phylogenetic analysis based on the sequences of the $5.8 \mathrm{~S}, 18 \mathrm{~S}$, and $28 \mathrm{~S}$ regions of the nrDNA confirmed the identity of the sequenced powdery mildew fungi as Pleochaeta shiraiana and Phyllactinia moricola. Both specimens belonged to strongly supported clades with reference specimens of their respective species (Fig. 1). In line with previous phylogenetic studies of the Erysiphaceae family (Bradshaw and Tobin 2020; Kiss et al. 2020; Liberato et al. 2006; Marmolejo et al. 2018; Takamatsu et al. 2016), powdery mildew species with hemiendophytic mycelium were clustered together in a clade with moderate phylogenetic support, with Pleochaeta shiraiana placed closer to Queirozia turbinata and Phyllactinia moricola showing a closer phylogenetic affinity to $L$. taurica.

In this announcement, we present the first draft genome resources for two powdery mildew fungi, Pleochaeta shiraiana and Phyllactinia moricola. Both powdery mildew species are little-studied pathogens of diverse tree species, with Pleochaeta shiraiana being common on Celtis spp. (Kiss et al. 2006) and Phyllactinia moricola on Morus spp. (Takamatsu et al. 2008). These represent two genera with hemiendophytic mycelium that have not yet been investigated with genomics tools. The evolutionary history of 


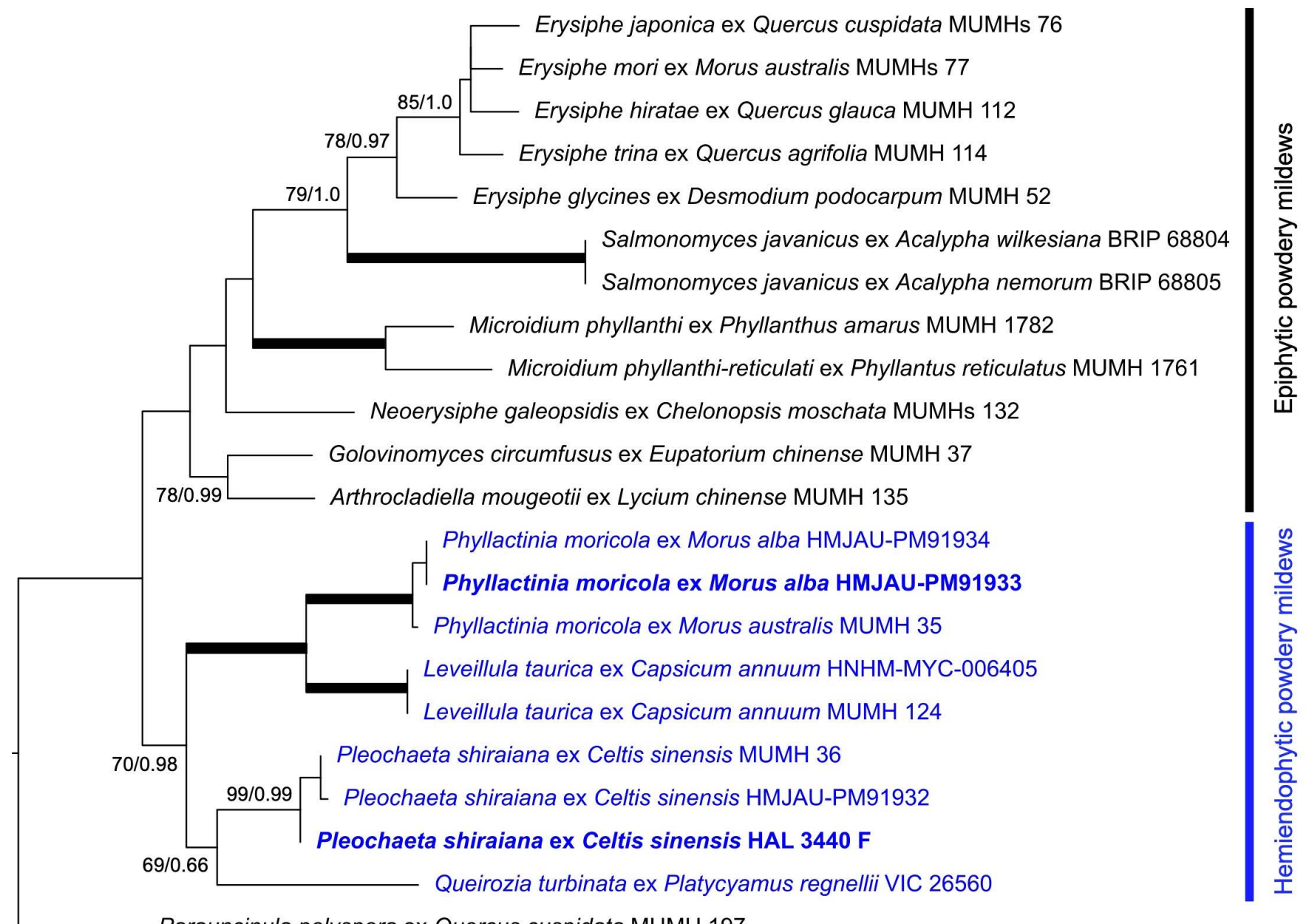

Parauncinula polyspora ex Quercus cuspidata MUMH 197

0.01

Fig. 1. Maximum-likelihood phylogram based on the concatenated sequences of the $5.8 \mathrm{~S}$, $18 \mathrm{~S}$, and $28 \mathrm{~S}$ regions of the nuclear ribosomal DNA of representative powdery mildew taxa. Maximum-likelihood bootstrap values (BS) and Bayesian Posterior Probability (PP) values are shown above or below the branches, separated by a slash. Thickened branches represent BS and PP values of $100 \%$ and 1.0 , respectively. Tip labels in bold represent specimens sequenced in the current study. The tree is rooted to Parauncinula polyspora MUMH 197, an early-diverged powdery mildew species (Frantzeskakis et al. 2019). The scale bar represents nucleotide substitutions per site.

tree-parasitic powdery mildew fungi is markedly different from herb-parasitic species (Takamatsu 2013). Therefore, the new genomic resources reported here will be highly valuable for future analyses toward understanding the differences between tree-parasitic and herb-parasitic species, as well as for deciphering the molecular basis of the unique lifestyle of powdery mildew fungi with hemiendophytic mycelium.

\section{Data Availability}

This Whole Genome Shotgun project has been deposited at NCBI GenBank under the accession PRJNA749662, and Sequence Read Archive accessions SRR15245358 (Pleochaeta shiraiana) and SRR15245359 (Phyllactinia moricola). The genome assemblies have been deposited at DNA Data Bank of Japan/European Nucleotide Archive/GenBank under the accessions JAHYSP000000000 (Pleochaeta shiraiana) and JAHYSQ000000000 (Phyllactinia moricola). The versions described in this article are versions JAHYSP010000000 and JAHYSQ010000000, respectively. The alignment and phylogenetic tree have been deposited in TreeBASE (number 28611).

\section{Acknowledgments}

We thank U. Braun (Martin-Luther-Universität Halle-Wittenberg, Germany) for his support with our Pleochaeta shiraiana herbarium specimen and J. Feng (Jilin Agricultural University, Changchun, China) for identifying Phyllactinia moricola samples. 


\section{Literature Cited}

Almagro Armenteros, J. J., Tsirigos, K. D., Sønderby, C. K., Petersen, T. N., Winther, O., Brunak, S., von Heijne, G., and Nielsen, H. 2019. SignalP 5.0 improves signal peptide predictions using deep neural networks. Nat. Biotechnol. 37:420-423.

Barsoum, M., Sabelleck, B., Spanu, P. D., and Panstruga, R. 2019. Rumble in the effector jungle: Candidate effector proteins in interactions of plants with powdery mildew and rust fungi. Crit. Rev. Plant Sci. 38:255-279.

Bindschedler, L., Panstruga, R., and Spanu, P. D. 2016. Mildew-Omics: How global analyses aid the understanding of life and evolution of powdery mildews. Front. Plant Sci. 7:123.

Bolger, A. M., Lohse, M., and Usadel, B. 2014. Trimmomatic: A flexible trimmer for Illumina sequence data. Bioinformatics 30:2114-2120.

Bradshaw, M., and Tobin, P. C. 2020. Sequencing herbarium specimens of a common detrimental plant disease (powdery mildew). Phytopathology 110:12481254.

Braun, U., and Cook, R. T. A. 2012. Taxonomic Manual of the Erysiphales (Powdery Mildews). CBS-KNAW Fungal Biodiversity Centre, Utrecht, The Netherlands.

Chan, P. P., Lin, B. Y., Mak, A. J., and Lowe, T. M. 2021. tRNAscan-SE 2.0: Improved detection and functional classification of transfer RNA genes. Nucleic Acids Res. 49:9077-9096.

Dutech, C., Feau, N., Lesur, I., Ehrenmann, F., Letellier, T., Li, B., Mouden, C., Guichoux, E., Desprez-Loustau, M. L., and Gross, A. 2020. An easy and robust method for isolation and validation of single-nucleotide polymorphic markers from a first Erysiphe alphitoides draft genome. Mycol. Prog. 19:615-628.

Feehan, J. M., Scheibel, K. E., Bourras, S., Underwood, W., Keller, B., and Somerville, S. C. 2017. Purification of high molecular weight genomic DNA from powdery mildew for long-read sequencing. J. Vis. Exp. 121:e55463.

Frantzeskakis, L., Kracher, B., Kusch, S., Yoshikawa-Maekawa, M., Bauer, S., Pedersen, C., Spanu, P. D., Maekawa, T., Schulze-Lefert, P., and Panstruga, R. 2018. Signatures of host specialization and a recent transposable element burst in the dynamic one-speed genome of the fungal barley powdery mildew pathogen. BMC Genomics 19:381.

Frantzeskakis, L., Németh, M. Z., Barsoum, M., Kusch, S., Kiss, L., Takamatsu, S., and Panstruga, R. 2019. The Parauncinula polyspora draft genome provides insights into patterns of gene erosion and genome expansion in powdery mildew fungi. MBio 10:381.

Glawe, D. A. 2008. The powdery mildews: A review of the world's most familiar (yet poorly known) plant pathogens. Annu. Rev. Phytopathol. 46:27-51.

Holt, C., and Yandell, M. 2011. MAKER2: An annotation pipeline and genomedatabase management tool for second-generation genome projects. BMC Bioinf. 12:491.

Jin, D.-N., Braun, U., Kiss, L., Takamatsu, S., Zhang, Z.-Y., de Álvarez, M. G. C., Li, Z., Li, Y., and Liu, S.-Y. 2021. Hidden diversity of powdery mildews belonging to the recently re-discovered genus Salmonomyces. Mycol. Prog. 20:10091018.

Jones, L., Riaz, S., Morales-Cruz, A., Amrine, K. C. H., McGuire, B., Gubler, W. D., Walker, M. A., and Cantu, D. 2014. Adaptive genomic structural variation in the grape powdery mildew pathogen, Erysiphe necator. BMC Genomics 15:1081.

Katoh, K., and Standley, D. M. 2013. MAFFT multiple sequence alignment software version 7: Improvements in performance and usability. Mol. Biol. Evol. 30:772780.

Kiss, L., Khosla, K., Jankovics, T., Niinomi, S., Braun, U., and Takamatsu, S. 2006. A morphologically ill-founded powdery mildew species, Pleochaeta indica, is recognized as a phylogenetic species based on the analysis of the nuclear ribosomal DNA sequences. Mycol. Res. 110:1301-1308.

Kiss, L., Vaghefi, N., Bransgrove, K., Dearnaley, J. D. W., Takamatsu, S., Tan, Y. P., Marston, C., Liu, S.-Y., Jin, D.-N., Adorada, D. L., Bailey, J., de Álvarez, M. G. C., Daly, A., Dirchwolf, P. M., Jones, L., Nguyen, T. D., Edwards, J., Ho, W., Kelly, L., Mintoff, S. J. L., Morrison, J., Németh, M. Z., Perkins, S., Shivas, R. G., Smith, R., Stuart, K., Southwell, R., Turaganivalu, U., Váczy, K. Z., van Blommestein, A., Wright, D., Young, A., and Braun, U. 2020. Australia: A continent without native powdery mildews? The first comprehensive catalog indicates recent introductions and multiple host range expansion events, and leads to the re-discovery of Salmonomyces as a new lineage of the Erysiphales. Front. Microbiol. 11:1571.

Kuhn, H., Kwaaitaal, M., Kusch, S., Acevedo-Garcia, J., Wu, H., and Panstruga, R. 2016. Biotrophy at its best: Novel findings and unsolved mysteries of the Arabidopsis-powdery mildew pathosystem. Arabidopsis Book 14:e0184.

Kusch, S., Németh, M. Z., Vaghefi, N., Ibrahim, H. M. M., Panstruga, R., and Kiss, L. 2020. A short-read genome assembly resource for Leveillula taurica causing powdery mildew disease of sweet pepper (Capsicum annuum). Mol. PlantMicrobe Interact. 33:782-786.

Laetsch, D. R., and Blaxter, M. L. 2017. BlobTools: Interrogation of genome assemblies. F1000 Res. 6:1287.

Liang, P., Liu, S., Xu, F., Jiang, S., Yan, J., He, Q., Liu, W., Lin, C., Zheng, F., Wang, X., and Miao, W. 2018. Powdery mildews are characterized by contracted carbohydrate metabolism and diverse effectors to adapt to obligate biotrophic lifestyle. Front. Microbiol. 9:3160.

Liberato, J. R., Barreto, R. W., Niinomi, S., and Takamatsu, S. 2006. Queirozia turbinata (Phyllactinieae, Erysiphaceae): A powdery mildew with a dematiaceous anamorph. Mycol. Res. 110:567-574.

Luo, R., Liu, B., Xie, Y., Li, Z., Huang, W., Yuan, J., He, G., Chen, Y., Pan, Q., Liu, Y., Tang, J., Wu, G., Zhang, H., Shi, Y., Liu, Y., Yu, C., Wang, B., Lu, Y., Han, C., Cheung, D. W., Yiu, S.-M., Peng, S., Xiaoqian, Z., Liu, G., Liao, X., Li, Y., Yang, H., Wang, J., Lam, T.-W., and Wang, J. 2012. SOAPdenovo2: An empirically improved memory-efficient short-read de novo assembler. Gigascience $1: 18$.

Marçais, G., and Kingsford, C. 2011. A fast, lock-free approach for efficient parallel counting of occurrences of k-mers. Bioinformatics 27:764-770.

Marmolejo, J., Siahaan, S. A. S., Takamatsu, S., and Braun, U. 2018. Three new records of powdery mildews found in Mexico with one genus and one new species proposed. Mycoscience 59:1-7.

Micali, C., Göllner, K., Humphry, M., Consonni, C., and Panstruga, R. 2008. The powdery mildew disease of Arabidopsis: A paradigm for the interaction between plants and biotrophic fungi. Arabidopsis Book 6:e0115.

Micali, C. O., Neumann, U., Grunewald, D., Panstruga, R., and O'Connell, R. J. 2011. Biogenesis of a specialized plant-fungal interface during host cell internalization of Golovinomyces orontii haustoria. Cell. Microbiol. 13:210-226.

Mohanta, T. K., and Bae, H. 2015. The diversity of fungal genome. Biol. Proced. Online 17:8

Müller, M. C., Kunz, L., Graf, J., Schudel, S., and Keller, B. 2021. Host adaptation through hybridization: Genome analysis of triticale powdery mildew reveals unique combination of lineage-specific effectors. bioRxiv. 06.442769.

Müller, M. C., Praz, C. R., Sotiropoulos, A. G., Menardo, F., Kunz, L., Schudel, S., Oberhänsli, S., Poretti, M., Wehrli, A., Bourras, S., Keller, B., and Wicker, T. 2019. A chromosome-scale genome assembly reveals a highly dynamic effector repertoire of wheat powdery mildew. New Phytol. 221:2176-2189.

Nylander, J. A. A. 2004. MrModeltest v2. Program distributed by the author. Evolutionary Biology Centre, Uppsala University, Uppsala, Sweden.

Panstruga, R., and Schulze-Lefert, P. 2002. Live and let live: Insights into powdery mildew disease and resistance. Mol. Plant Pathol. 3:495-502.

Ronquist, F., and Huelsenbeck, J. P. 2003. MrBayes 3: Bayesian phylogenetic inference under mixed models. Bioinformatics 19:1572-1574.

Simão, F. A., Waterhouse, R. M., loannidis, P., Kriventseva, E., and Zdobnov, E. M. 2015. BUSCO: Assessing genome assembly and annotation completeness with single-copy orthologs. Bioinformatics 31:3210-3212.

Smit, A. F. A., Hubley, R., and Green, P. 2016. RepeatMasker Open-4.0. 20132015. https://www.repeatmasker.org/

Spanu, P. D., Abbott, J. C., Amselem, J., Burgis, T. A., Soanes, D. M., Stüber, K., van Themaat, E. V. L., Brown, J. K. M., Butcher, S. A., Gurr, S. J., Lebrun, M.-H., Ridout, C. J., Schulze-Lefert, P., Talbot, N. J., Ahmadinejad, N., Ametz, C., Barton, G. R., Benjdia, M., Bidzinski, P., Bindschedler, L., Both, M., Brewer, M. T., Cadle-Davidson, L., Cadle-Davidson, M. M., Collemare, J., Cramer, R., Frenkel, O., Godfrey, D., Harriman, J., Hoede, C., King, B. C., Klages, S., Kleemann, J., Knoll, D., Koti, P. S., Kreplak, J., López-Ruiz, F. J., Lu, X., Maekawa, T., Mahanil, S., Micali, C. O., Milgroom, M. G., Montana, G., Noir, S., O'Connell, R. J., Oberhänsli, S., Parlange, F., Pedersen, C., Quesneville, H., Reinhardt, R., Rott, M., Sacristán, S., Schmidt, S. M., Schön, M., Skamnioti, P., Sommer, H., Stephens, A. E., Takahara, H., Thordal-Christensen, H., Vigouroux, M., Weßling, R., Wicker, T., and Panstruga, R. 2010. Genome expansion and gene loss in powdery mildew fungi reveal tradeoffs in extreme parasitism. Science 330:1543-1546.

Sperschneider, J., and Dodds, P. N. 2021. EffectorP 3.0: Prediction of apoplastic and cytoplasmic effectors in fungi and oomycetes. Mol. Plant-Microbe Interact.

Stamatakis, A. 2014. RAxML version 8: A tool for phylogenetic analysis and postanalysis of large phylogenies. Bioinformatics 30:1312-1313.

Stanke, M., and Waack, S. 2003. Gene prediction with a hidden Markov model and a new intron submodel. Bioinformatics :ii215-ii225.

Takamatsu, S. 2013. Molecular phylogeny reveals phenotypic evolution of powdery mildews (Erysiphales, Ascomycota). J. Gen. Plant Pathol. 79:218-226.

Takamatsu, S., Inagaki, M., Niinomi, S., Khodaparast, S. A., Shin, H.-D., Grigaliunaite, B., and Havrylenko, M. 2008. Comprehensive molecular phylogenetic analysis and 
evolution of the genus Phyllactinia (Ascomycota: Erysiphales) and its allied genera. Mycol. Res. 112:299-315.

Takamatsu, S., Siahaan, S. A. S., Moreno-Rico, O., Cabrera de Álvarez, M. G., and Braun, U. 2016. Early evolution of endoparasitic group in powdery mildews: Molecular phylogeny suggests missing link between Phyllactinia and Leveillula. Mycologia 108:837-850.

Wicker, T., Oberhänsli, S., Parlange, F., Buchmann, J. P., Shatalina, M., Roffler, S., Ben-David, R., Doležel, J., Šimková, H., Schulze-Lefert, P., Spanu, P. D., Bruggmann, R., Amselem, J., Quesneville, H., van Themaat, E. V. L., Paape, T.,
Shimizu, K. K., and Keller, B. 2013. The wheat powdery mildew genome shows the unique evolution of an obligate biotroph. Nat. Genet. 45:1092-1096.

Wu, Y., Ma, X., Pan, Z., Kale, S. D., Song, Y., King, H., Zhang, Q., Presley, C., Deng, X., Wei, C.-I., and Xiao, S. 2018. Comparative genome analyses reveal sequence features reflecting distinct modes of host-adaptation between dicot and monocot powdery mildew. BMC Genomics 19:705.

Zheng, Z., Nonomura, T., Bóka, K., Matsuda, Y., Visser, R. G. F., Toyoda, H., Kiss, L., and Bai, Y. 2013. Detection and quantification of Leveillula taurica growth in pepper leaves. Phytopathology 103:623-632. 